\title{
Optical Detection and Spectroscopy of Single Molecules in a Solid
}

\author{
W. E. Moerner and L. Kador ${ }^{(a)}$ \\ IBM Research Division, Almaden Research Center, San Jose, California 95120
}

(Received 17 March 1989)

\begin{abstract}
Using two different double-modulation techniques, we have observed the optical-absorption spectrum of single dopant molecules of pentacene in a $p$-terphenyl host crystal at liquid-helium temperatures. To achieve this, frequency-modulation spectroscopy was combined either with Stark or ultrasonic modulation to remove interfering background signals from residual amplitude modulation, and the number of molecules in resonance was reduced to one by operating in the wings of the inhomogeneous line. Triplet bottleneck saturation appears to be suppressed in the single-molecule regime.
\end{abstract}

PACS numbers: $78.50 .-\mathrm{w}, 33.20 . \mathrm{Kf}, 33.70 . \mathrm{Jg}$

There has been much recent interest in optical detection and spectroscopy of single absorbers in various environments. Single atomic ions in vacuum confined in electromagnetic traps have been detected and cooled and have shown a variety of interesting quantum jump ${ }^{1}$ and photon antibunching phenomena ${ }^{2}$ that test our understanding of quantum physics. In liquid media, single viruses and bacteria ${ }^{3}$ and single proteins with multiple chromophores ${ }^{4}$ have been detected using optical traps and hydrodynamic focusing techniques, respectively.

The detection of single absorbers in a solid would provide an important new tool for the study of local absorber-host interactions that would be uncomplicated by the normal averaging over as many as $10^{4}$ to $10^{16}$ similar absorbers. Furthermore, in a solid at liquidhelium temperatures the absorbing centers are effectively trapped by the host matrix and therefore show no Doppler or recoil effects. In fluorescence experiments, the problem of detecting the signal from a single absorber in a transparent host is complicated by Rayleigh and/or Raman background signals from the large number $\left(10^{10}-10^{18}\right)$ of host molecules or ions in the probing volume. To date, the fluorescence from as few as five absorbing ions in a crystal at $77 \mathrm{~K}$ has been detected using a fixed frequency laser. ${ }^{5}$ Far more information about the line shape of a single absorber and the possible perturbations due to the local environment could be obtained if the actual frequency-dependent absorption spectrum of a single absorbing center in a solid could be recorded.

Recent measurements of statistical fine structure (SFS) on an inhomogeneously broadened optical transition of pentacene in $p$-terphenyl crystals ${ }^{6,7}$ provide an alternative approach to single-molecule detection (SMD) and spectroscopy. The SFS experiments used a powerful, low-background technique, laser frequencymodulation (FM) spectroscopy, ${ }^{8}$ to detect only the variations in the absorption coefficient on a frequency scale less than the modulating frequency $v_{m}$; in this regime SFS scales as $\left(\bar{N}_{H}\right)^{1 / 2}$, where $\bar{N}_{H}$ is the average number of absorbers in the probed volume within one homogene- ous linewidth. The observations of SFS were performed near the inhomogeneous line center with relatively large values of $\bar{N}_{H}\left(\simeq 10^{5}\right)$. To achieve SMD, we proceed to the $\bar{N}_{H}<1$ limit by moving out in laser wavelength to the wings of the inhomogeneous line where $\bar{N}_{H}$ can be made arbitrarily small. ${ }^{9}$ For example, if one assumes a Gaussian shape for the inhomogeneous line with a standard deviation of $0.02 \mathrm{~nm}$ [full width at half maximum (FWHM) $42 \mathrm{GHz},{ }^{10}$ then the SMD spectra reported below were observed at a distance from the line center equal to 6-8 standard deviations. The value of $\bar{N}_{H}$ at the line center was approximately $10^{3}-10^{4}$ in our samples and thus in the SMD region the simple Gaussian model would predict $\bar{N}_{H}=10^{-8}-10^{-7}$. It is not too surprising that there are long tails on the inhomogeneous line that extend out further than predicted by the Gaussian model, corresponding to more and more improbable, highly strained sites.

Although FM spectroscopy is quantum limited in principle, the true sensitivity limit in many cases is controlled by interfering background signals from residual amplitude modulation (RAM). ${ }^{11}$ To overcome this, we utilized two new techniques: FM Stark double modulation $(\text { FMS })^{12}$ and FM ultrasound double modulation (FMUS). Both methods rely on direct internal modulation of the absorption line with an external field and a second demodulation step to remove RAM.

The measurements were performed on the inhomogeneously broadened $\mathrm{O}_{1}(592.32 \mathrm{~nm})$ and $\mathrm{O}_{2}(592.18$ $\mathrm{nm})$ site zero-phonon origin absorptions of pentacene dopant molecules in Bridgman-grown $p$-terphenyl single crystals ${ }^{10}$ immersed in superfluid helium at $1.6 \mathrm{~K}$. Single-molecule signals were observed on both the red edge of $\mathrm{O}_{1}$ and the blue edge of $\mathrm{O}_{2}$, although only the long-wavelength results will be described here. Total concentrations ranged between $1 \times 10^{-6}$ and $2 \times 10^{-7}$ $\mathrm{mol} / \mathrm{mol}$, and the measurements were performed on cleaved samples $100-200 \mu \mathrm{m}$ in thickness. To prevent thermal drift of the focal volume during acquisition of the spectra, a small 0.85 numerical aperture lens of $4.2-$ $\mathrm{mm}$ focal length on an electromagnetic positioner was 
attached directly to the sample holder in the liquid helium. The tunable rhodamine- $6 \mathrm{G}$ dye laser $(\simeq 3 \mathrm{MHz}$ linewidth) was typically set to scan over $1 \mathrm{GHz}$ in $0.25 \mathrm{~s}$; hence the molecule was probed in the quasi-steady-state limit (i.e., the passage time of the laser through the molecular absorption line was longer than any molecular relaxation time). Full details of the experimental apparatus will be presented elsewhere. ${ }^{13}$

An important advantage of FM spectroscopy ${ }^{14}$ is that the detected signal approaches zero for signals from any background molecules with linewidths much larger than $v_{m}$. The linewidth for pentacene is less than or equal to our values of $v_{m}$, so the FM signal for one molecule is proportional to $(\Delta \alpha) L=\sigma / A$, where $\Delta \alpha$ is the difference in optical absorption at the upper and lower optical sidebands, $L$ is the sample thickness, $\sigma$ is the peak lowtemperature absorption cross section, and $A$ is the crosssectional beam area. For our focal spot diameter of $\simeq 3$ $\mu \mathrm{m}$, and neglecting any possible power broadening effects, the expected signal from a single pentacene $a b-$ sorber at the focus would be $(\Delta \alpha) L \simeq 1 \times 10^{-4}$. Detection of such signals with FM techniques is improved when RAM is suppressed. ${ }^{11}$

The first three traces in Fig. 1 show simulations of the

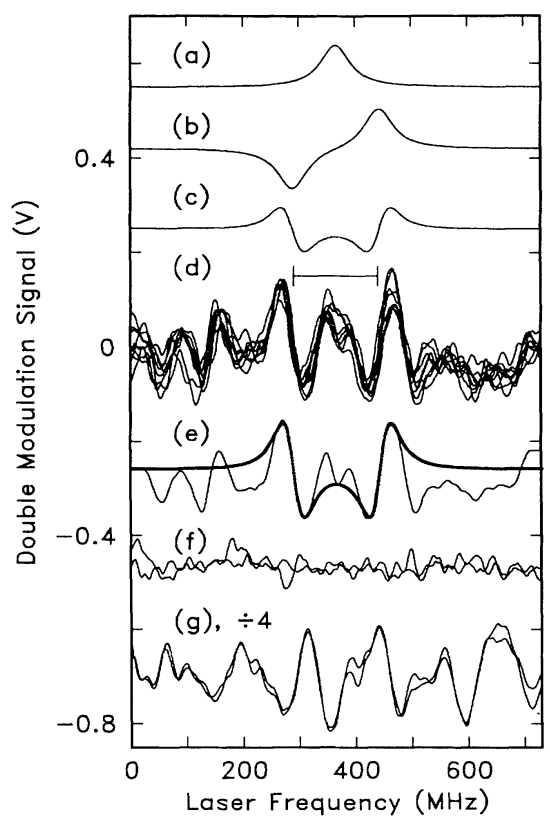

FIG. 1. Illustration of single-molecule spectra using FMS technique. (a) Simulation of absorption line, $\gamma=65 \mathrm{MHz}$. (b) Simulation of FM spectrum for (a), $v_{m}=75 \mathrm{MHz}$. (c) Simulation of FMS line shape. (d) SMD spectra at $592.423 \mathrm{~nm}$, 512 averages, 8 traces overlaid, bar shows value of $2 v_{m}=150$ $\mathrm{MHz}$. (e) Average of traces in (d) $\left(\mathrm{S}_{2}\right.$ removed) with fit to the in-focus molecule (smooth curve). (f) Signal far off line at $597.514 \mathrm{~nm}$. (g) Traces of SFS at the $\mathrm{O}_{2}$ line center, 592.186 $\mathrm{nm}$. expected single-molecule line shape for either Stark or ultrasonic double modulation. Figure 1(a) shows the usual Lorentzian absorption profile $L_{\gamma}(v)=(\gamma / 2 \pi) /$ $\left[v^{2}+(\gamma / 2)^{2}\right]$, where $\gamma$ (FWHM) has been set equal to $65 \mathrm{MHz}$ for comparison purposes. When $v_{m} \gg \gamma$, simple FM detection (in the absorption, or $S_{1}$, phase ${ }^{14}$ ) produces a line shape composed of two copies of the absorption profile of opposite sign, spaced by $2 v_{m}$ [Fig. 1(b)]. With secondary modulation (at frequencies $\ll v_{m}$ and $\ll \gamma$ ), the center frequency of the original Lorentzian profile oscillates and the subsequent time dependence in the FM signal of Fig. 1(b) is detected with a phasesensitive detector. The resulting line shape (for smallfrequency deviations) is the first derivative of the FM spectrum as shown in Fig. 1(c). Thus the essential signature of SMD is a large negative slope and a large positive slope separated by $2 v_{m}$.

For the FMS measurements, the samples were sandwiched between two indium-tin-oxide-coated sapphire disks for application of a sinusoidal Stark field at 5 $\mathrm{kHz}$ with peak value $45 \mathrm{kV} / \mathrm{cm}$. Since pentacene has a quadratic Stark effect, ${ }^{15}$ final demodulation was performed with a lock-in amplifier at $10 \mathrm{kHz}(1.25 \mathrm{~ms}$ time constant). Figure 1(d) shows a set of eight FMS double-modulation spectra of a strong in-focus molecule far out in the long-wavelength edge of $\mathrm{O}_{1}$, along with several unavoidable weak spectra of out-of-focus molecules at the left and right edges of the laser scan range. The fiducial bar marks the value of $2 v_{m}$. The expected " $W$ " line shape is clearly visible, with the exception of a larger-than-expected increase in signal at the center of the line. This central distortion is most likely due to a weak contribution from the molecular dispersion $\left(S_{2}\right.$ phase ${ }^{14}$ ) and can be explicitly removed, as has been done in Fig. 1(e) for the average of the eight scans of Fig. 1(d). Also shown in Fig. 1(e) is a fit to the strong central feature using a simple theory for the FMS technique. $^{13}$ The fit to the major features of the strong infocus molecule is reasonable. However, the value of $\gamma$ required by the fitting process, $65 \mathrm{MHz}$, is larger than the photon-echo value $\left(\simeq 8 \mathrm{MHz}^{16}\right)$ due possibly to local field fluctuations (see below), the detection time constant, or other effects. Because of signal-to-noise ratio (SNR) limitations, our main goal in this study was to detect the major features of the single-molecule spectrum rather than to unequivocally determine the linewidth.

Although absolute calibrations of zero-background spectra are difficult, important insight comes from an approximate comparison of the amplitude of the central $W$ in Fig. 1(d) with that expected for a single molecule. By calibration of the simple FM signal with a known absorption, $1 \mathrm{~V}$ on the vertical scale in Fig. 1 corresponds to an absorbance change $(\Delta \alpha) L=1.8 \times 10^{-3}$, if the Stark shift is on the order of $\gamma$. The absorbance amplitude of the observed SMD signal of $\simeq 1.8 \times 10^{-4}$ is com- 
patible with the expected value, if power broadening is not severe. In our experiments, we adjusted the light power on the avalanche photodiode detector to $\simeq 0.3$ $\mu \mathrm{W}$ in order to obtain a favorable SNR. The resulting sideband intensity of $\simeq 1 \mathrm{~W} / \mathrm{cm}^{2}$ is much larger than the saturation intensity of pentacene including triplet saturation, ${ }^{13} 70 \mathrm{~mW} / \mathrm{cm}^{2}$. The fact that we nevertheless see approximately the full signal can be explained if triplet bottleneck effects are suppressed. The triplet yield has been shown to vary among the major sites of pentacene in $p$-terphenyl, ${ }^{16}$ and here we have evidence that molecules in the wings of the inhomogeneous line for the $\mathrm{O}_{1}$ site may have reduced intersystem crossing.

Figure 1(f) shows spectra from a wavelength so far away from the pentacene site origins that no molecules are expected to lie in the laser scan range; this is the background shot and avalanche noise. In samples of undoped pure $p$-terphenyl, only a base line noise level similar to the off-line data in Fig. 1(f) was observed, even near the center of the inhomogeneous line.

Figure 1(g) shows spectra of SFS near the inhomogeneous line center for the $\mathrm{O}_{2}$ site (for convenience) using a smaller number of averages. This spectrum is composed of a superposition of many $W$ profiles similar to Fig. 1(d) with many different center frequencies, illustrating the qualitative difference between spectra of large

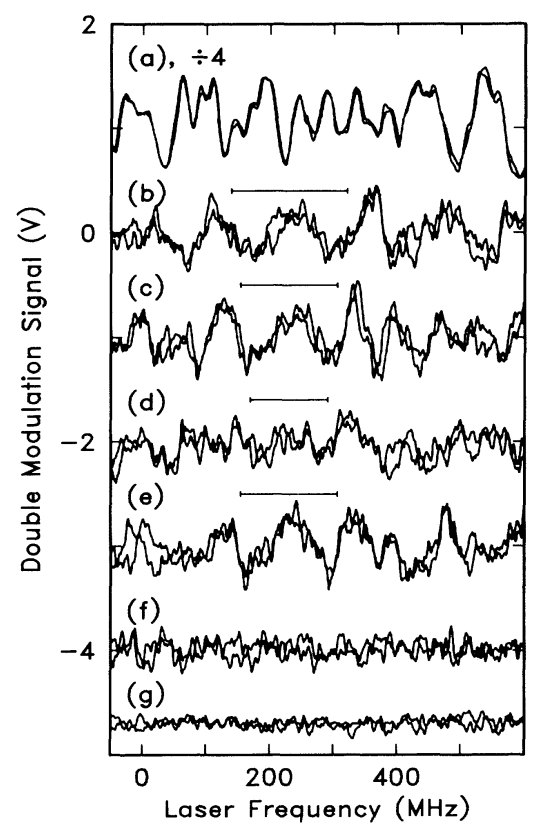

FIG. 2. Examples of single-molecule spectra using the FMUS technique. (a) Traces of SFS at the $\mathrm{O}_{1}$ line center, $592.312 \mathrm{~nm}, 128$ averages each. (b) SMD spectra at 592.469 nm, $v_{m}=91 \mathrm{MHz}, 128$ averages, bar shows the value of $2 v_{m}$. (c) $v_{m}=76 \mathrm{MHz}$. (d) $v_{m}=61 \mathrm{MHz}$. (e) Back to $v_{m}=76$ $\mathrm{MHz}$. (f) Far off line at $590.462 \mathrm{~nm}$. (g) No light on the detector. numbers of molecules [Fig. 1(g)] and SMD [Fig. 1(d)]. The SFS is only $\approx 4$ times larger than the SMD signals due mainly to triplet bottleneck saturation at the center of the inhomogeneous line.

We observed spectra similar to Fig. 1(d) on several occasions with several different samples; however, it was difficult to reliably observe SMD spectra each day with the FMS technique. Furthermore, with smaller Stark fields, the SFS near line center became somewhat narrower (and weaker). We believe that these effects may be due to a well-known effect in molecular crystals: ${ }^{17}$ charge injection into the $p$-terphenyl crystal during the detection process and subsequent time-dependent variations in the local electric field at the pentacene sites.

To overcome the problems with high electric fields, we also recorded spectra with the newly developed FMUS technique, which is essentially a combination of FM spectroscopy and an ultrasonic modulation method reported previously for the detection of spectral holes. ${ }^{18}$ Since in this case the molecular absorption line is shifted by an applied stress field, charge motion and injection become unimportant. The samples were bonded to a transparent shear-mode quartz transducer driven at 2 $\mathrm{MHz}$ and final demodulation was performed phase sensitively at the same frequency. The expected lineshapes for FMUS are the same as for FMS detection.

Figure 2(a) shows that FMUS can produce strong repeatable spectra of SFS at the $\mathrm{O}_{1}$ line center. Far out in the wings of the inhomogeneous line, single-molecule features are also observed [Figs. 2(b)-2(e)]. For example, in Fig. 2(b) taken with $v_{m}=91 \mathrm{MHz}$, the expected $W$ structure appears near the center of the trace, with signals from out-of-focus molecules at other locations within the scan. For both techniques, we found it difficult to record spectra with only one strong in-focus molecule and no out-of-focus molecules due simply to the fact that the Rayleigh range of the laser focal spot was much smaller than the sample thickness. The apparent linewidth $(\simeq 40 \mathrm{MHz})$ is smaller than with FMS, but still larger than the previously measured homogeneous width due perhaps to ultrasonic mode conversion or other effects.

In Figs. 2(c), 2(d), and 2(e), the value of $v_{m}$ was changed to 76,61 , and back to $76 \mathrm{MHz}$, respectively. The $W$ line shape clearly expands and contracts as expected in order to maintain the distance between the large negative and large positive slopes equal to $2 v_{m}$. Such clear variations of the line shape with $v_{m}$ can only occur for a single molecule (or for two or more molecules with exactly the same center frequency, but this is unlikely to occur so far out in the wings of the inhomogeneous line). When many molecules are present as in Fig. $2(\mathrm{a})$, the signal is a superposition of many $W$ line shapes with different center frequencies, and well-defined changes with $v_{m}$ are not observed.

The fact that similar SMD spectra were observed with 
two radically different external fields rules out a number of artifacts that might arise from only one of these methods. This, in conjunction with the shape and approximate size of the features, the dependence on $v_{m}$, the position relative to the inhomogeneous line center, and our insensitivity to any features wider than $\simeq 100 \mathrm{MHz}$ from nonrigid impurities, leads us to conclude that the spectra reported here are due to single molecules of pentacene in $p$-terphenyl. Thus, the $\bar{N}_{H}=1$ limit of SFS in inhomogeneously broadened lines in solids has been attained. Future development of techniques for recording SMD spectra at lower intensity and higher SNR should allow additional study of single local environments in solids without averaging over large numbers of "equivalent" molecular configurations. Such work would open up a new frontier of spectroscopy of single defect centers in solids where no Doppler, recoil, or multicenter averaging effects are present.

We acknowledge early groundwork by T. P. Carter, stimulating discussions with G. C. Bjorklund, and crucial electronic design assistance from D. E. Horne. This work was supported in part by the U.S. Office of Naval Research and the IBM World Trade organization.

(a) Present address: Department of Experimental Physics, University of Bayreuth, Bayreuth, West Germany.

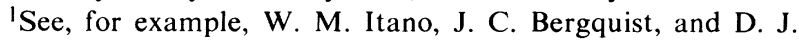
Wineland, Science 237, 612 (1987), and references therein.
${ }^{2}$ F. Diedrich, J. Krause, G. Rempe, M. O. Scully, and H. Walther, IEEE J. Quantum Electron. 24, 1314 (1988).

${ }^{3}$ A. Ashkin and J. M. Dziedzic, Science 235, 1517 (1987).

${ }^{4}$ D. C. Nguyen, R. A. Keller, J. H. Jett, and J. C. Martin, Anal. Chem. 59, 2158 (1987).

${ }^{5}$ R. Lange, W. Grill, and W. Martienssen, Europhys. Lett. 6, 499 (1988).

${ }^{6}$ W. E. Moerner and T. P. Carter Phys. Rev. Lett. 59, 2705 (1987).

${ }^{7}$ T. P. Carter, M. Manavi, and W. E. Moerner, J. Chem. Phys. 89, 1768 (1988).

${ }^{8}$ G. C. Bjorklund, Opt. Lett. 5, 15 (1980).

${ }^{9}$ W. E. Moerner, Bull. Am. Phys. Soc. 34, 656 (1989).

${ }^{10}$ R. W. Olson and M. D. Fayer, J. Phys. Chem. 84, 2001 (1980).

${ }^{11}$ E. A. Whittaker, M. Gehrtz, and G. C. Bjorklund, J. Opt. Soc. Am. B 2, 1320 (1985).

${ }^{12}$ T. P. Carter, D. E. Horne, and W. E. Moerner, Chem. Phys. Lett. 151, 102 (1988).

${ }^{13}$ L. Kador, D. E. Horne, and W. E. Moerner (to be published).

${ }^{14}$ G. C. Bjorklund, M. D. Levenson, W. Lenth, and C. Ortiz, Appl. Phys. B 32, 145 (1983).

${ }^{15}$ J. H. Meyling and D. A. Wiersma, Chem. Phys. Lett. 20, 383 (1973).

${ }^{16}$ F. G. Patterson, H. W. H. Lee, L. Wilson, and M. D. Fayer, Chem. Phys. 84, 51 (1984).

${ }^{17}$ F. P. Chen, S. J. Sheng, and D. M. Hanson, Chem. Phys. 5, 60 (1974).

${ }^{18}$ W. E. Moerner and A. L. Huston, Appl. Phys. Lett. 48, 1181 (1986). 